\title{
Central venous catheter guidewire salvage technique to prevent a never event
}

\section{Mariyaselvam M. ${ }^{1}$,Richardson $\mathrm{J}^{2}$, Sawyer A. ${ }^{2}$ and Young P. ${ }^{2}$}

1. Department of Anaesthesiology and Intensive Care, University of Cambridge, 2. The Critical Care Department, The Queen Elizabeth Hospital Kings Lynn NHS Foundation Trust

\section{Background and Goal of Study:}

Accidental guidewire retention following central venous catheter (CVC) insertion is the 2nd commonest reported retained foreign object in the UK and is a never event. The NHS England National Reporting and Learning System (NRLS) database 2004-2015 reported 239 guidewire retentions of which on only one occasion was suction reportedly applied to the distal lumen prior to removal. If recognised early, the guidewire is more likely to be still in the catheter lumen. A common technique for guidewire removal is to clamp the catheter at the skin and pull the assembly back. However, if the wire has passed beyond the skin level, this is likely to be ineffective. We tested a rapid suctioning prior to clamping to improve the chances of success.

\section{Materials and Methods:}

Following IRB approval, we used a bench model with a guidewire placed $2 \mathrm{~cm}$ into the distal tip of a CVC catheter simulating a wire about to embolise. The $\mathrm{CVC}$ was positioned in a quarter circle conformation and the tip and guidewire was submerged in a water bath simulating the circulation. A $20 \mathrm{ml}$ syringe was attached to the distal lumen and strong suction was continuously applied for $5 \mathrm{~s}$ by vigorously retracting the plunger and guidewire retraction measured. We tested $3 \mathrm{CVCs}, 2$ vascaths and 2 wide-bore introducers 10 times each. Success was determined by the wire moving back into the catheter thereby improving the chances of clamping and removal.

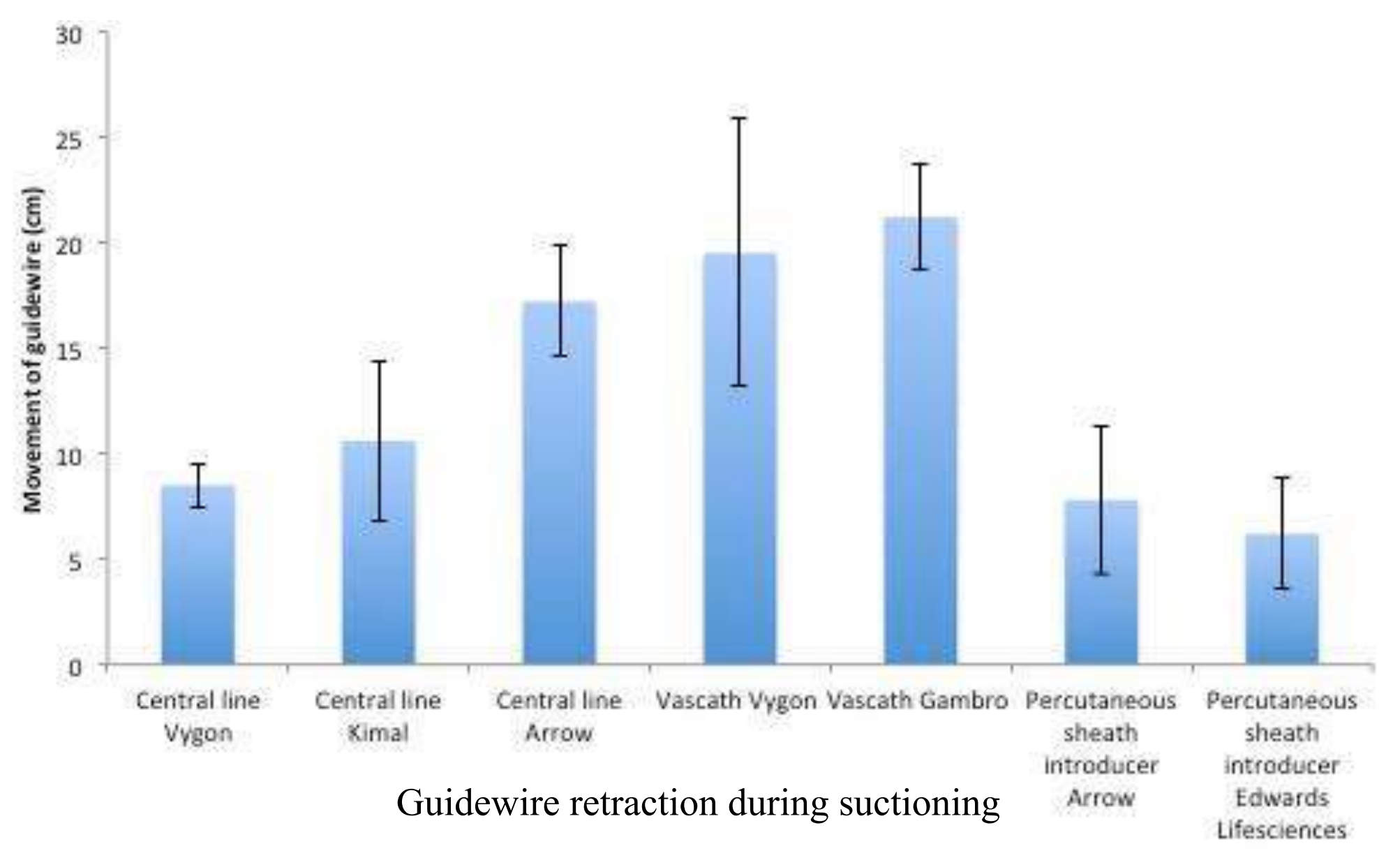

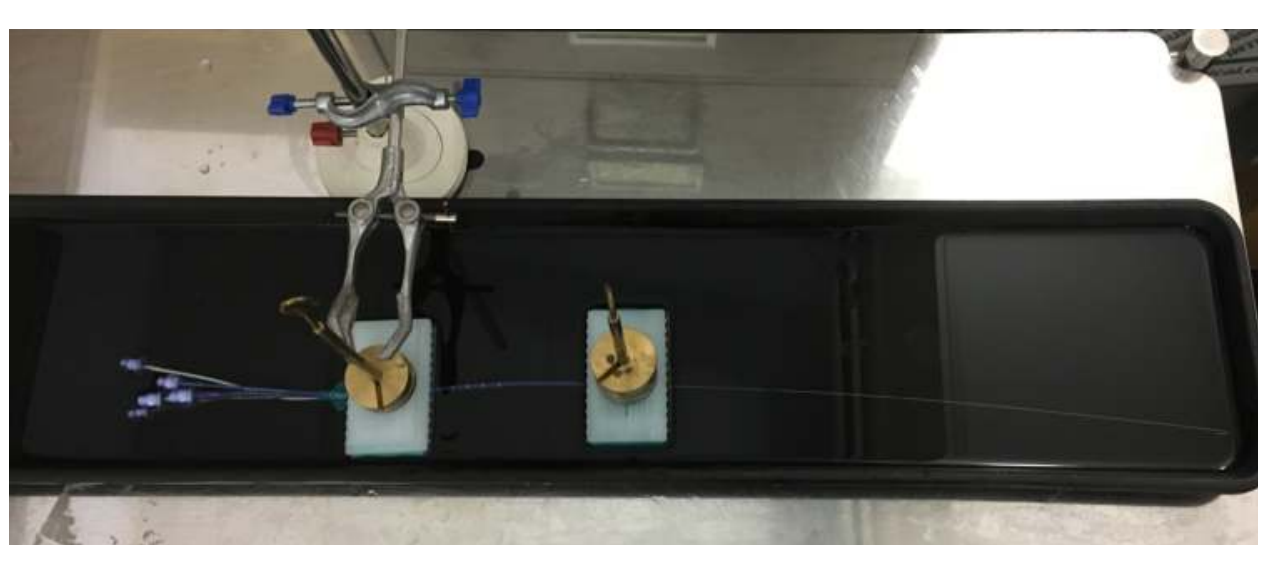

Benchtop model and apparatus used

\section{Results and Discussion:}

The rapid suck technique successfully retracted the wire in all scenarios and for all catheters (graph shows means and $95 \% \mathrm{CI}$ ).

Only the Vascaths, the wide bore catheters and the Arrow CVC reliably retracted the wire beyond the estimated skin level.

\section{Conclusions:}

The intraluminal position of a retained guidewire may be unknown, may be difficult to see on $\mathrm{x}$-ray, and delays or patient manipulation may cause further migration.

If there has been a decision to remove the catheter then suction should be applied first to retract the wire.

Further simulation studies should examine whether following rapid suction, additional clamping or continuous suctioning on catheter removal is more effective for guidewire retrieval.

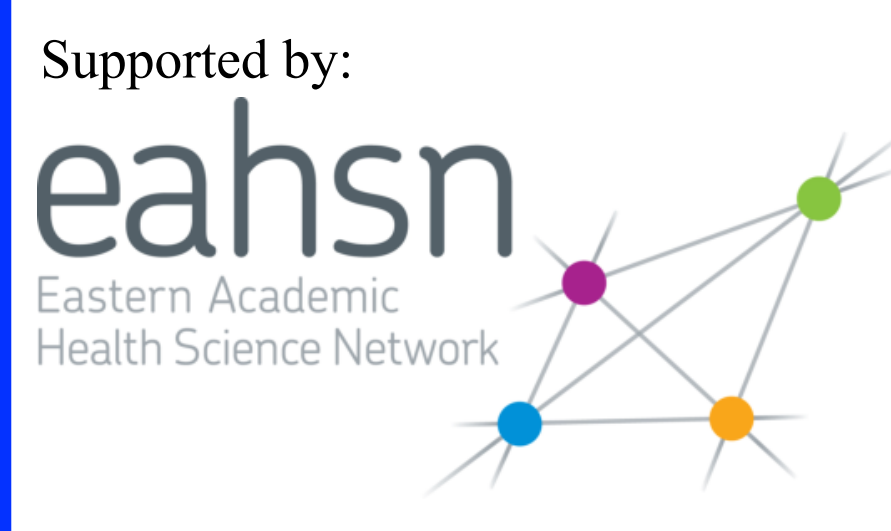

